\title{
Correction to: Tetsugaku Companion to Japanese Ethics and Technology
}

\author{
Thomas Taro Lennerfors and Murata Kiyoshi
}

\section{Correction to:}

Thomas Taro Lennerfors, Murata Kiyoshi (eds.),

Tetsugaku Companion to Japanese Ethics and Technology, Tetsugaku Companions to Japanese Philosophy 1, https://doi.org/10.1007/978-3-319-59027-1

This book was inadvertently published with the incorrect font (Adobe Myungjo Std) used to render Japanese and Chinese characters. This font has now been changed to MS Mincho throughout the book except in Chapter 6 where original Chinese characters are changed to SimSun font. 